\title{
Epistemología de la imposibilidad o ciencia de la indeterminación
}

\section{Epistemology of the impossible or a science of indetermination}

\author{
Carlos Eduardo Maldonado (maldonadocarlos@unbosque.edu.co) Facultad de Medicina, \\ Universidad El Bosque (Bogotá, Colombia) https://orcid.org/0000-0002-9262-8879
}

\begin{abstract}
This paper starts with a question and is devoted to solving a problem, namely so establish whether an epistemology of the impossible or a science of indetermination are at all possible. In order to solve the problem three arguments are set out: firstly, impossibility very much as indetermination are not to be conceived in any sense as limits, restraints or constrictions. Quite on the contrary, they are assets that are won or acquired in the field of research and knowledge. The second argument claims that the impossible entails abandoning the entire tradition centred around being, reality, the given, what happens or what is at-hand. What is at stake is a fantastic scientific revolution. The third argument point out that the world and the universe do not have any specific determination, and certainly not one established beforehand. Based on these arguments this paper argues that there is not disjunction between an epistemology of the impossible and a science of indetermination, for research can go forwards thanks to discovery or invention which are a twofold form of research and education that know about change and metamorphosis. Several conclusions are drawn at the end vis-à-vis the social and human sciences.
\end{abstract}

Key words: complexity, non-linearity, non-monotonicity, meta-theory, epistemology.

\section{Resumen}

Este artículo parte de un interrogante y se propone abordar un problema, a saber, si existen y son posibles una epistemología de la imposibilidad o, lo que es equivalente, una ciencia de la indeterminación. A fin de resolver el problema, se proponen tres argumentos. Esto son: primero, la imposibilidad, como la indeterminación, no deben ser concebido en modo alguno como limitaciones, restricciones o carencias. Por el contrario, se trata de ganancias o adquisiciones en el campo del conocimiento y de la investigación. En segundo término, la imposibilidad significa un abandono de toda la tradición centrada en la importancia del ser, la realidad, lo dado, lo que acaece o lo que está ahí. Lo que está en juego es una fantástica revolución científica. El tercer argumento pone en evidencia que el mundo y el universo no tienen una determinación específica, y ciertamente no dada de antemano. Sobre la base de estos tres argumentos el artículo afirma que no hay ninguna disyuntiva entre una epistemología de la imposibilidad y una ciencia de la indeterminación, y que la buena investigación puede avanzar por este camino gracias al descubrimiento o la invención, que es la dúplice forma de una educación e investigación que sabe de cambios y transformaciones. Algunas conclusiones de cara a las ciencias sociales y humanas se extraen el final.

Palabras clave: complejidad, no-linealidad, no-monotonicidad, metateoría, epistemología. 


\section{Introducción}

A partir del poema de Parménides, la tradición occidental es ciencia, filosofía y cultura de lo real, de lo que acaece, lo que está ahí, en fin, lo que sucede. La forma categorial como esto se expresa es que se trata de ciencia y filosofía del ser: tò gàr aútò noein éstín te kaì einai (pues son lo mismo pensar y ser). De consuno, como enseña la tradición, el no ser no es, y si fuera, no lo podríamos pensar, y si lo pudiéramos pensar no lo podríamos decir (Gorgias); en fin, nada entra al ser que no sea el ser, y nada sale del ser que no sea el ser (Tomás de Aquino, Hegel). En esta identificación se condensan dos mil quinientos años de civilización occidental. La epistemología y la ontología son, en esta tradición, una sola y misma cosa. Así, el filósofo y el científico -para el caso no hay ninguna diferencia- están abocados a pensar lo que es, y nada por fuera suyo, punto.

Es apenas en el giro del siglo XIX al siglo XX cuando se comienza a pensar no ya única y principalmente acerca de la realidad, sino además y fundamentalmente, acerca de la posibilidad. La puerta de acceso grande a lo posible se denomina espacio de Hilbert, pero encuentra su antecedente más importante en la teoría de conjuntos formulada por Cantor. El nacimiento de las lógicas no-clásicas en general, y de la lógica modal y multimodal en particular, termina de allanar el camino, y se abre un panorama amplio, dicho en general, gracias al modelamiento y la simulación; es decir, gracias a ese instrumento cultural que es el computador y la computación. Sería un tema aparte hacer la historia del descubrimiento de lo posible, un capítulo apasionante, sin duda, en la historia de la ciencia.

Las ciencias de la complejidad introducen una inflexión importante en la ecología del conocimiento puesto que hacen de lo posible el ámbito específico de su trabajo, sus investigaciones y desarrollos. Sin ambages, las ciencias de la complejidad son ciencias de posibilidades. Por ello mismo, el lenguaje que las caracteriza, tal como "espacio de configuración", "espacio de fases", "transiciones de primer orden y de segundo orden", "estados críticos", "puntos críticos", "criticalidad autoorganizada", el trabajo con modelamiento y simulación, su especificidad por el trabajo con metaheurísticas, el criterio diferencial de las leyes de potencia, y otros rasgos adicionales.

Es posible afirmar esta inflexión en torno al estudio, aprehensión y creación de posibilidades de esta manera: en el curso de la segunda mitad del siglo XX hasta la fecha, hacemos ciencia de los efectos; no ya, por primera vez, de las causas. Entonces se habla genéricamente, por ejemplo, de efectos cuánticos, consecuencias de la existencia y comportamiento de un agujero negro, consecuencias del fracking y la crisis climática, las consecuencias del agotamiento de los hidrocarburos, los efectos de las ondas gravitacionales o de las explosiones de supernovas, y muchos más.

La ciencia de lo real tiene una preocupación principal. Se trata de las anticipaciones, las tendencias, los vectores o los desarrollos probables de lo que hay y sucede. Por esta razón emergen diversas estrategias de análisis como la prospectiva, el análisis de riesgos, los estudios de tendencias, y otros semejantes. La gran dificultad con esa clase de ciencia es que no sabe de sorpresas, de contingencias, de excepciones. Tan solo de procesos generales y universales. Jamás pudo, como sucedió efectivamente, haber previsto acontecimientos singulares, como el martes negro de 1929, el ascenso y triunfo de Hitler en 1933, la caída del muro de Berlín en 1989, la elección de un papa jesuita argentino y muchos más. De hecho, la historia está llena de excepcionalidades. La metáfora recientemente acuñada para designar esta clase de fenómenos y sistemas es la de cisne negro; pero el concepto que en propiedad le pertenece es el de eventos raros. 
Pues bien, el siglo XX y XXI ha hecho el descubrimiento, aún más anodino, de la imposibilidad, y ha hecho ciencia de ella. Es a partir de este reconocimiento que emerge el título de este trabajo: ¿es posible una epistemología de la imposibilidad, o bien, cabe pensar en ciencia (y filosofía) de la indeterminación? Este es el problema que define a este artículo y ésta también su novedad.

Este texto se propone exponer y dilucidar el problema formulado. Para ello, se avanza en tres pasos estratégicos. En primer lugar, se elabora el parangón entre imposibilidad (o indeterminación) y ciencia de los límites; sin recursos retóricos, se trata acaso igualmente de la consideración cuidadosa acerca de los límites de la ciencia. Esta es la primera sección de este estudio. La segunda sección se detiene en el significado propio de la imposibilidad y muestra los ejemplos más destacados de cómo efectivamente existe una ciencia de la imposibilidad y qué comporta ello. Sobre esta base, la tercera sección se ocupa de la indeterminación y plantea un tema eminentemente epistemológico, a saber: ¿la indeterminación es inventada o descubierta? y se resuelve el interrogante. Las conclusiones presentan la tesis de este estudio y afirma que tenemos ante nosotros, con nosotros, efectivamente, una epistemología de la imposibilidad o una ciencia (y filosofía) de la indeterminación y explica en qué consisten ambas.

Este artículo constituye la continuación de una línea de investigación, algunos de cuyos antecedentes son la consideración de la emergencia de ciencias sociales irregulares (Maldonado. Ciencias sociales irregulares), la metamorfosis de las ciencias sociales, el procesamiento noalgorítmico de la información, en fin, la importancia de los eventos raros en la epistemología y la complejidad. Las ciencias sociales constituyen la verdadera piedra de toque de la epistemología, pues en ellas los temas de contrastación, falseabilidad criterios de demarcación y muchos más son, al final del día, un asunto de vida o muerte; bastante, por tanto, más que un mero juego académico o intelectual.

En verdad, los asuntos relativos a la epistemología se traducen al final del día en cuestiones relativas a condiciones posibilitantes o amenazantes y negativas de vida. La epistemología en general está lejos de ser un asunto neutro en la ecología del conocimiento.

\section{Ciencia de límites, límites de la ciencia e imposibilidad}

Vivimos el cruce y las tensiones de tres revoluciones científicas. Inicialmente, la primera revolución científica, la ciencia de la modernidad, que hoy ha perdido su fuerza revolucionaria y se ha convertido en ciencia normal. Luego, la revolución cuántica que tiene todavía una fuerza enorme en numerosos dominios de la sociedad y cuyo cénit verosímilmente se encuentra aún lejano. Y, en tercer lugar, la teoría de la información, la cual ha llegado a entrecruzarse con la cuántica dando lugar al procesamiento cuántico de información, con todos los desarrollos en torno a la computación y la supremacía cuánticas, la impronta de la inteligencia y de la vida artificial, la web 3.0 actual y las siguientes, ya previsibles, web 4.0 y la 5.0, el sentido y el aprovechamiento de la web profunda, por lo pronto. La cuarta revolución industrial en curso constituye una de las facetas más claras a escala cotidiana de las revoluciones en marcha.

Pues bien, precisamente en medio de este panorama emerge, una y otra vez, con intereses y acentos distintos, la idea del surgimiento del límite de la ciencia, tanto como la posibilidad de una ciencia de los límites. La bibliografía al respecto es amplia y creciente. En realidad, estas no son sino modos de abordaje de conflictos profundos entre estructuras mentales -con todo y sus cargas 
económicas, políticas y sociales- entre las tres revoluciones científicas. Ante este panorama hay voces críticas y pesimistas de un lado, tanto como optimistas y de vanguardia de otra parte. La sociología de la ciencia, los estudios culturales sobre la ciencia y la tecnología, y la propia historia de la ciencia conoce una amplia bibliografía de un lado y del otro. Los límites de la ciencia están sottovoce referidos a las limitaciones e incapacidades de la ciencia clásica. Se trata, en otras palabras, de todas las anomalías presentes en la ciencia moderna, para decirlo en el lenguaje de Kuhn.

Pues bien, es posible condensar estas tensiones y conflictos en términos de lógica o de matemáticas. Se trata de las transiciones entre lo actual, lo necesario y lo posible; por ejemplo, se trata de las (a)simetrías entre hipótesis (lo posible), contrastaciones (lo actual) y demostraciones (lo necesario). Así las cosas, cabría pensar en algo así como ontologías o epistemologías transitorias en el sentido de A. Badiou.

Es evidente que la ciencia clásica o moderna -esto es, más exactamente, la primera revolución científica- ya perdió la carga emancipatoria que alguna vez tuvo. Con ella es posible aún hacer cosas, efectivamente; pero ya perdió la capacidad explicativa o comprensiva que alguna vez alcanzó. Así, la idea del límite de la ciencia debe ser leída inevitablemente con respecto a la ciencia moderna, es decir, aquella que, si cabe decirlo en términos puntuales, comienza con F. Bacon, con R. Bacon, con Galileo y se proyecta hasta Einstein, pasando por Descartes, Vesalius, Newton, Smith, Ricardo, Koch, Pasteur y Comte, entre otros. Es a esta clase de ciencia a la que los acontecimientos asaltan sorpresivamente. Es con respecto a ella que bien cabe la idea de Hegel acerca del Búho de Minerva: que emprende su vuelo cuando cae la tarde. En verdad, lo mejor que puede hacer esa ciencia son predicciones retrospectivas, que es, hoy por hoy, una forma de hacer mala ciencia.

Pues bien, el descubrimiento de la posibilidad como un tema propio; y a fortiori, el descubrimiento de la imposibilidad, no debe ser asimilado, para nada, a algo semejante a una ciencia de límites, como tampoco a los límites de la ciencia. Una analogía se impone, por ejemplo, a propósito del llamado principio de incertidumbre de Heisenberg: con él, no perdemos para nada las certezas que alguna vez alcanzamos; además, ganamos la incertidumbre. Pues bien, el descubrimiento y tematización gradual de lo posible y lo imposible constituyen ganancias que marcan una profunda inflexión en la historia del conocimiento humano, por lo menos desde la Grecia antigua.

En otros términos, lo que alguna vez fuera el ser -para decirlo en clave filosófica- no se erosiona para nada, sino que se inscribe en un marco, más amplio y profundo, que lo comprende y lo hace posible, a saber: el marco de la posibilidad. Y ulteriormente, en el marco aún más extenso y sugestivo de la imposibilidad. Sucede a la manera como la geometría euclidiana se convierte en un caso particular de las geometrías no-euclidianas, como la mecánica clásica se convierte en un caso particular de la mecánica relativista. No perdemos para nada ninguna acepción de lo real (lo actual, lo que es, lo que viene al caso, lo dado, y demás), sino, se lo inscribe en el ámbito bastante más amplio y profundo de lo posible. Por numerosos caminos, la ciencia y la filosofía del siglo XX son el descubrimiento de la posibilidad como una dimensión con estatuto propio: la matemática, la lógica, la física cuántica y la física de partículas, la astronomía y la cosmología, la bioquímica y la biología sintética; pero también la filosofía (Husserl y Deleuze), la antropología (Pandian), la sociología (Ofshe y Epstein), por ejemplo. En verdad, pensar el ser o la realidad es importante, pero es demasiado poco. Además, y fundamentalmente, debemos poder pensar en posibilidades, y con ellas 
y a través de ellas, en imposibilidades. Al cabo, ulteriormente, se trata de saber de, y trabajar con, indeterminaciones.

El trabajo con posibilidades, imposibilidades e indeterminaciones consiste simple y llanamente en la exploración de eventualidades, de eventos raros, el estudio de colas largas, acontecimientos altamente improbables, y demás. De manera herramental, si cabe decirlo así, el modelamiento y la simulación han contribuido a este descubrimiento y al trabajo con los mismos. Pero la verdad es que el modelamiento y la simulación no son otra cosa que la instrumentalización computacional de la conditio sine qua non de la buena ciencia y filosofía, a saber: el trabajo con experimentos mentales, el uso de la imaginación y la fantasía, las pompas de intuición.

Recientemente, en especial a partir del año 2010, a lo anterior se agrega la ciencia de grandes bases de dato (big-data science) y por tanto la analítica de datos. Modelamiento y simulación y la analítica de datos consisten esencialmente en el aprendizaje de, y el trabajo con, lenguajes de programación y sus fortalezas (estadística, interfaces y cruces entre lenguajes distintos, la escritura de códigos, y otros rasgos semejantes). No sin datos, la buena investigación es además y fundamentalmente, creación y/o discusión de mundos posibles, y à la limite, de mundos imposibles.

Digamos que, en ciencia, como en la vida, siempre que se produce un vaivén entre lo real y lo ideal, entre lo fáctico y lo posible, entre la realidad y la ficción, en fin, entre lo inmediato y lo imaginable. Así las cosas, es preciso combinar ambos extremos sin reducir el asunto, y ciertamente no a priori, a cualquier de los extremos. La complejidad del conocimiento y la investigación emerge a partir de los juegos de contraposición y contrapunto entre lo real, lo necesario y lo posible. La simple consideración del tipo "en algún momento en el pasado" o "en algún punto en el futuro", introducidos por la lógica del tiempo de Prior sirve para ilustrar la idea en cuestión. Las posibilidades constituyen modalizaciones de un estado cosas dado, y consisten en el llamado a juegos ideatorios o pompas de intuición que no son, en absoluto, encuentros o choques con límites, sino, mucho mejor, exploración de dimensiones y espacios de fase.

Otra manera de entender que el trabajo con posibilidades no es, en absoluto, el choque con limitaciones, restricciones o carencias es mediante la puesta de manifiesto que buena parte de lo mejor de la investigación de punta en el mundo es ciencia de efectos o consecuencias, no ya simplemente de causas. Sin la menor duda, el surgimiento de enormes bases de datos ha permitido identificar tan rápidamente las causas de numerosos fenómenos y sistemas, que la atención se ha derivado, consecuentemente, hacia el estudio de las consecuencias o los efectos. Un excelente ejemplo al respecto es el surgimiento del posibilismo en geografía y en estudios ambientales en general, el cual surge como un ejemplo conspicuo en favor del argumento de este artículo.

Subsecuentemente, la racionalidad científica en general ha aprendido acerca de la importancia de las correlaciones; esto es, el hecho, literal, de que los efectos pueden ser a su vez causas de las causas, sin que haya circularidad. Los estudios de correlaciones ponen sobre la mesa, a plena luz del día, la complejidad de las investigaciones contemporáneas y la ingenuidad del pasado, consagrado estrictamente al estudio de la causalidad; que era cuando había poca información disponible y entonces se hablaba de variables: dependientes, independientes, diversos tipos de variables aleatorias, moderadoras, extrañas y otras. En el marco del modelamiento y la simulación y con mayor razón en el contexto de la analítica de datos (big-data science), "variable" se ha convertido, 
sencillamente, en una palabra, sin más, y toda la atención se vuelva sobre correlaciones, emergencias y redes.

Pues bien, el estudio de los efectos o consecuencias implica la apertura hacia posibilidades o eventualidades, contingencias e indeterminaciones. Pues, por definición, los efectos son impredecibles e incontrolables. $Y$ debe ser posible consiguientemente hacer buena ciencia de efectos. Esto es lo que denominamos ciencias de posibilidades, cuya heurística en general incorpora experimentos mentales, el uso de la fantasía, la exploración de posibles adyacentes y otros aspectos próximos y semejantes.

Cuando la investigación se ocupa de fenómenos variables o evolutivos, oscilatorios, marcados por fluctuaciones, inestabilidades y turbulencias y en escenarios de redes complejas (esto es, redes de mundo pequeño, redes libres de escala o redes aleatorias), entre otros atributos, lo necesario y actual se revela como inconstante invitando así a una explicación de sus posibles desarrollos. En verdad, estos desarrollos posibles nada tienen que ver con el espacio local (euclidiano) y remiten, consiguientemente, a espacios de posibilidades. Asistimos así, entonces, a una revolución en la estructura mental (mindset) de trabajo, experimentación, relacionamiento o intervención del mundo y/o la sociedad.

De esta suerte, efectos o consecuencias y posibilidades pueden ser aprehendidos como dos fases de una sola dinámica que entonces puede ser adecuadamente llamada como no-lineal. Nunca huelga subrayar este aspecto: un sistema lineal es aquel que gana información, aunque no necesariamente por ello gane memoria. Que un sistema gane memoria se dice en metateoría o en lógicas no-clásicas como no-monotonicidad.

\section{¿Qué significa la imposibilidad?}

El tema de base es el pensar o la investigación (aquí pueden ser tomados por lo pronto como equivalentes). Y nada ni nadie puede restringir al pensar libre o a la buena investigación crítica a un ámbito determinado, a un parámetro preestablecido, en fin, a un marco rígido, cualquiera que sea. La historia de la ciencia, la filosofía y la investigación en general puede ser vista como la emancipación del realismo y la ganancia de las posibilidades, en toda la línea de la palabra. Todo ello sucede cuando las doctrinas han colapsado o han dejado de ser hegemónicas.

Cuando la investigación se ocupa de estructuras, dinámicas o fenómenos cambiantes, inestables, impredecibles y no-lineales, más vale ampliar los marcos de referencia, las formas de organización mental, estar abiertos a eventualidades, no despreciar de entrada ninguna probabilidad y reconocer que cualquier cosa puede suceder. Pues bien, es exactamente esto, dicho de modo general, lo que comporta aprender acerca de la imposibilidad.

Nadie piensa bien si no piensa en todas las posibilidades, e incluso en lo imposible mismo; esto es, literalmente, en lo inaudito, inopinado, inverosímil, improbable, y demás. La imposibilidad existe y la buena investigación debe poder ganarla. No es, en absoluto, como se aprecia, un límite o una restricción, sino una ganancia. Pues bien, existe una gama sólida de reconocimientos de que es posible una ciencia de lo imposible. El cuadro No. 1 presenta los ejes principales de una ciencia semejante. 
Cuadro No. 1: Ejes principales de una ciencia de lo imposible

\begin{tabular}{|c|c|c|}
\hline Origen & Modo & Autores \\
\hline Matemáticas, lógica y filosofía & $\begin{array}{l}\text { Paradojas, como la del Hotel } \\
\text { Infinito de Hilbert, o el abuelo } \\
\text { y la máquina de viajar en el } \\
\text { tiempo, o de Russell }\end{array}$ & Hilbert, Peano, Russell. \\
\hline Lógica & $\begin{array}{l}\text { Teorema de la incompletitud } \\
\text { de Gödel }\end{array}$ & Gödel \\
\hline Física cuántica & $\begin{array}{l}\text { Principio de indeterminación } \\
\text { de Heisenberg }\end{array}$ & Heisenberg \\
\hline Economía & $\begin{array}{l}\text { Teorema de imposibilidad de } \\
\text { Arrow }\end{array}$ & Arrow \\
\hline $\begin{array}{l}\text { Matemáticas, topología, } \\
\text { teoría de homologías }\end{array}$ & Cohomología & Kolmogorov, Smale, Penrose \\
\hline $\begin{array}{l}\text { Teoría de las probabilidades, } \\
\text { estadística, complejidad }\end{array}$ & Cisnes negros & Taleb \\
\hline $\begin{array}{l}\text { Teoría de la complejidad } \\
\text { computacional }\end{array}$ & $\begin{array}{l}\text { Problemas indecidibles, en el } \\
\text { marco de la teoría de la } \\
\text { complejidad computacional }\end{array}$ & Turing, Karp, Cook, Levin \\
\hline $\begin{array}{l}\text { Teoría de la computación, } \\
\text { hipercomputación }\end{array}$ & $\begin{array}{l}\text { Cualquier máquina que no } \\
\text { obedezca la tesis Church- } \\
\text { Turing }\end{array}$ & Church, Turing, Syropoulos \\
\hline $\begin{array}{l}\text { Ciencias de la complejidad, } \\
\text { teoría de la computación }\end{array}$ & Hipercomputación biológica & $\begin{array}{l}\text { Burgin, Cooper, Maldonado, } \\
\text { Gómez-Cruz }\end{array}$ \\
\hline $\begin{array}{l}\text { Matemáticas, estadística, } \\
\text { teoría del valor extremo }\end{array}$ & $\begin{array}{l}\text { La transición de eventos raros } \\
\text { a eventos imposibles }\end{array}$ & King, Zheng, Maldonado \\
\hline
\end{tabular}

Fuente: Maldonado. Las ciencias de la complejidad son ciencias de la vida.

Como se aprecia, se trata de ciencia -matemáticas, lógica, computación, física cuántica, ciencias de la complejidad, estadística, economía, notablemente- que aparecen específicamente en el siglo XX y XXI. En ningún caso se trata de algo así como descartar posibilidades, sino por el contrario, en el reconocimiento expreso de que existe la imposibilidad y hay que contar con ella. Así, por ejemplo, es imposible que un sistema democrático de elección cumpla al mismo tiempo con las cinco condiciones señaladas en su teorema por parte de Arrow; o bien, es imposible establecer al mismo tiempo el lugar y el momento de una partícula, como en Heisenberg; o también, es imposible que un sistema deductivo formal sea consistente y completo al mismo tiempo como en Gödel, para mencionar tan solo los ejemplos más conocidos.

La imposibilidad aquí desborda ampliamente cualquier consideración de tipo biográfica, sexual, económica, cultural, histórica o psicológica por parte de algún agente o grupo o colectividad. En una palabra, la imposibilidad es ontológica, por así decirlo.

Las matemáticas contemporáneas (aquellas que abarcan desde mediados del siglo XX hasta la fecha), han hecho el descubrimiento de que existen estructuras y objetos (matemáticos) imposibles, y que hay que trabajar con ellos. Tal es, dicho sucintamente, el objeto de la cohomología. Esto 
significa el desafío para pensar contraintuitivamente, pues la percepción natural descansa siempre sobre actualidades y facticidad; es este todo el mundo que se designa gramaticalmente en el modo del indicativo. Este es el mundo del sentido común, de lo dado, lo que acaece, lo que está a la mano (vorhanden-sein y zuhanden sein, en Heidegger), el ser-ahí (dasein), en fin, todo aquello que está arrojado en el mundo (geworfen), para decirlo de manera gráfica.

Subrayemos este rasgo cultural: lo posible y lo imposible no existen en un espacio euclidiano, y como tal, no se los ve con los ojos ni descansan en la percepción natural. Una epistemología de lo imposible o una ciencia de la indeterminación no coinciden para nada con la cultura, en el sentido primero de la palabra, esto es, con atavismos, costumbres y hábitos. La posibilidad, la imposibilidad y la indeterminación deben ser construidos, esto es, ganados, simulados, adquiridos, conquistados, que no son sino diferentes maneras de plantear que se trata de adquisiciones y no de encuentros como puntos de partida. En el ámbito de las ciencias sociales, se trata de toda la dimensión compuesta por utopías, sueños, programas, futuros, eventualidades, contingencias, impredecibilidad, en fin, literalmente: complejidad. De lejos, el más crítico de los temas referentes a la impredecibilidad es el medioambiente; y con él, claro, la crisis climática. "Humans may have developed conditional adaptions that enabled accelerated life history development if exposed to environmental unpredictability" (Young et al. 2020:551).

La carga cultural de lo que precede no escapa a un entendimiento sensible. En las conclusiones volvemos sobre esta idea. Hay que precisar que las discusiones acerca del constructivismo y el construccionismo no entran aquí en consideración. Si lo posible y lo imposible existen, deben ser descubiertos, o bien, inventados. El tema hace referencia a las formas de pensamiento tanto como a las herramientas de la investigación. Vuelvo sobre este tema en la siguiente sección.

\section{El encuentro de la indeterminación}

El mundo no tiene una determinación específica, y ciertamente no una a pesar de otras. Literalmente, el universo y la realidad no tienen una forma determinada, y no puede ser explicado de forma determinista sin caer por ello, al mismo tiempo, en el reduccionismo. Así las cosas, la indeterminación constituye uno de los rasgos propios de la vida y el mundo, cuando se los ve sin prejuicios.

Precisemos una cosa. Debemos poder hablar de indeterminación (gracias a Heisenberg), aunque en la historia reciente quizás el primer antecedente se encuentra en el apeiron de Anaximandro, que puede ser entendido al mismo tiempo como lo indefinido, el infinito, lo indeterminado, y no ya simplemente incertidumbre. (Digamos entre paréntesis, que Anaximandro tiene la excepcionalidad que, junto a Heráclito, jamás formaron parte del guion canónico occidental, lo cual es un motivo suficiente para volver la mirada, una vez más, sobre ellos).

Cuando Heisenberg formula el principio de indeterminación en el famoso artículo de 1927 Über den anschaulichen Inhalt der quantentheoretischen Kinematik und Mekanik (Acerca del contenido intuitivo de la cinemática y la mecánica de la teoría cuántica), emplea dos términos de manera indistinta para hablar de la imposibilidad de identificar al mismo tiempo la posición y el movimiento de una partícula. Estos dos términos son Ungenauigkeit y Unbestimmtheit. El primero significa imprecisión, y el segundo, indeterminación. En ningún momento usa la palabra Unsicherheit, que significa incertidumbre, y que sí aparece, por lo demás, en otro contexto, en la obra de Z. Bauman. 
La traducción de Unbestimmtheit por "incertidumbre" es equivocada y se presta, como ha sido efectivamente el caso en numerosas ocasiones, a interpretaciones subjetivistas. Así las cosas, el mal llamado "principio de incertidumbre" debe ser adecuadamente entendido como principio de indeterminación. Los errores parecen haber comenzado por la traducción al inglés de la idea de Heisenberg. De todos los ejes presentados en el cuadro No. 1, sin la menor duda la contribución de Heisenberg es la más acendrada en la cultura académica y científica. Por ello, la necesidad de esta precisión puntual.

El mundo, de entrada, aparece como indeterminado. La historia del espíritu humano es el proceso mediante el cual: a) le adscribimos formas y determinaciones particulares; b) unas formas son sustituidas por otras en una historia interminable que comprende a la historia del teatro, de la pintura, de la música, de la poesía, de la filosofía y de la ciencia, principalmente. La historia del mundo y el universo es, así, una sola con la historia del espíritu humano. Solo que, en un marco más amplio, esta es la historia misma de procesos de codificación y decodificación que se encuentra en la base misma de la vida y de la materia y que son el objeto de la biosemiótica. Por razones de espacio debemos dejar de lado, sin embargo, una ampliación de la biosemiótica, y referir sencillamente a los trabajos seminales de Sebeok, Hoffmeyer, Wheeler, Thom y Kauffman, principalmente.

Como se observa, la posibilidad y la imposibilidad forman parte de los procesos mismos de transformación de la realidad, en cada momento y caso. Pero, la indeterminación ¿es invención o descubrimiento? Esta pregunta requiere una consideración propia.

Los temas que se encuentran en entredicho con el interrogante formulado hacen referencia a las relaciones entre el idealismo ontológico y el idealismo epistemológico, el realismo ontológico y el realismo epistemológico, para decirlo en términos clásicos.

Pues bien, la tesis de este estudio afirma que la indeterminación ( $\mathrm{y}$ a fortiori, también la imposibilidad), forman parte de un lugar fronterizo entre invención y descubrimiento. Pensar en fronteras no es sino una manera de hablar de intersticios, comisuras, pliegues, transiciones, catástrofes, todos términos propios de la complejidad del conocimiento y del mundo y el universo.

Una característica fundamental de lo mejor de la buena investigación de punta en el mundo hoy (spearhead science) es que ha aprendido a moverse entre territorios diversos sin caer por ello en el relativismo o el eclecticismo, y que ha aprendido por tanto acerca de la importancia de procesos (por definición abiertos y de largo alcance), así como sobre la necesidad de transiciones y adaptaciones; en fin, está alcanzando gradualmente el reconocimiento acerca del importancia y la necesidad de elaborar síntesis. Podemos decirlo entonces de una dúplice manera: o bien la determinación de la vida y el mundo es simplemente parcial y temporal (lo cual hace pensar en los jardines zen, por ejemplo), o bien el universo y el mundo carecen de determinación alguna y deben poder ser aprehendidos como tales; esto es, deben poder aprehendidos como indeterminación. De cara a la tradición occidental esto parece imposible. QED, sostendría un matemático.

Lo que está en juego con la indeterminación es por consiguiente la creatividad del espíritu humano o bien, lo que es equivalente, el carácter esencialmente abierto y evolutivo de la vida en general. Así las cosas, una sana inteligencia tanto como una sensibilidad refinada combinan invención y descubrimiento, y no tienen por qué asumirlas en términos de jerarquías o prioridades. Las fluxiones 
de la inteligencia y de la vida saben de movimientos, transformaciones y adaptaciones incesantes, lo cual pone de manifiesto que la invención y el descubrimiento son complementarios. Esta complementariedad orienta la mirada hacia las dimensiones de la posibilidad, la imposibilidad y la indeterminación. El mundo en general se aparece entonces más amplio, profundo y rico de lo que jamás pudo haber sucedido antes en la historia, particularmente si se lo mira con los ojos de la civilización occidental.

Una epistemología de la imposibilidad tanto como una ciencia de la indeterminación saben de libertad, esto es, específicamente, de grados de libertad, y entonces, claro, de vida. Que es lo único que importa al final del día. Es decir, horizontes, posibilidades abiertas, inconclusiones, aperturas inacabadas. Ser occidentales significa creer que las cosas tienen comienzo, y que como tienen comienzo entonces tienen igualmente final. Una creencia semejante pierde todos sus pivotes a la luz de una epistemología de posibilidades de imposibilidades y de indeterminaciones.

\section{Conclusión}

El más importante y difícil de todos los problemas en buena ciencia y filosofía consiste en distinguir lo trivial de lo no-trivial, algo que se dice fácilmente, pero que es arduo de llevar a cabo. Por ejemplo: es trivial elaborar enunciados universales o generales; es trivial hacer uso de herramientas y técnicas ya disponibles; en fin, es trivial establecer inferencias directas. Por vía de contraste, es no-trivial elaborar enunciados particulares o singulares, y el empleo entonces de cuantificadores particulares; es no-trivial desarrollar nuevas herramientas, técnicas, lenguajes y metodologías frente a los nuevos temas, objetos y problemas que se descubren y con los que se trabaja subsiguientemente; es notrivial trabajar con inferencias indirectas, inferencias inductivas y transductivas. Pues bien, este texto argumenta que partir de la realidad y remitirse a ella al final del día es trivial. La buena ciencia y filosofía se mueven de lo conocido hacia lo desconocido; aunque resulte tautológico, la buena educación, información, investigación, ciencia y filosofía ni permanecen en la esfera de lo conocido ni se mueven en dirección suya.

Por el contrario, es la exploración de posibilidades, la consideración incluso de imposibilidades es lo que caracteriza a la buena investigación. Y ello comporta un rasgo extremadamente difícil, a saber: tener una estructura de mente abierta. La verdad es que la mayoría de las gentes no tienen una estructura de mente abierta justamente porque poseen ya un sexo, una lengua materna, unas condiciones socio-económicas, acaso un credo, un pasado, y demás. Para ellos, el mundo acaece y si acaso, después, se explica.

Pues bien, la realidad, con todos sus rasgos y atributos, es trivial. La historia del espíritu humano consiste en superarla, en enriquecerla, en hacerla cada vez mejor, en negarla incluso, en fin, en transformarla, de tantas maneras como sea imaginable. Tal es la historia del arte, la educación, la ciencia y la filosofía, dicho de forma genérica. Y entonces, una sensibilidad aguda, una imaginación nutrida, una existencia angustiada, por ejemplo, le introducen a la realidad lo que ella no tiene: posibilidades, e incluso imposibilidades. Gramaticalmente, se trata, frente el imperio del modo del indicativo, de otros modos; por ejemplo, el modo del condicional, o el modo del subjuntivo. (Claro, esto, en el marco de los idiomas indoeuropeos).

La realidad solo se sabe a sí misma, y solo se quiere a sí misma. Una traducción de esta idea es la institucionalidad y el neo-institucionalismo (político, económico, sociológico, y otros) los cuales se 
definen como negación de posibilidades y de imposibilidades. El término en el que se condensa una epistemología y ciencia semejantes es, hoy por hoy, como la afirmación y la defensa del status quo, que no es sino un eufemismo para decir: el ser (to on hêi on, tanto como el tò tí ên eînai, según Aristóteles). Lo cierto es que el mundo no está determinado, y no posee una forma más que otra, y ciertamente no una forma establecida a priori a pesar de otra(s).

El título de este artículo anuncia una disyuntiva. Pues bien, el problema se resuelve eliminando el "o". En otras palabras, tenemos hoy, por primera vez, una epistemología de la imposibilidad o, lo que es equivalente, una ciencia de indeterminación. Culturalmente hablando, se trata de un campo pequeño e incipiente, pero la razón tiene que ver con las dimensiones de la información y la educación. Precisamente en este sentido aparece, en toda su radicalidad, la idea de revolución en el conocimiento, que no es sino la expresión abstracta para decir una revolución en la vida, con todas las implicaciones, traducciones y matices que ello comporta.

Tenemos ante nosotros, anticipados por algunos ejes referenciales, una epistemología de la imposibilidad (que comprende a una epistemología de lo posible), o también, una ciencia de la indeterminación. Una epistemología semejante concuerda con la idea de ciencias irregulares, de eventos raros, de procesamiento no-algorítmico de la información y con una profunda transformación de las ciencias y disciplinas.

Un horizonte generoso y amplio emerge ante la mirada. La epistemología no tiene por qué ser un asunto adusto y grave, acaso à la Popper; al fin y al cabo, la marca de calidad de la vida está en el cambio, en las transformaciones, en los procesos; que no es sino una manera, genérica, de hablar de posibilidades, de indeterminaciones y de pensar y vivir en términos de imposibilidades. Pues esta esfera de la imposibilidad es tanto inventada como descubierta. Todo depende, según parece, de las disposiciones y capacidades del investigador o del grupo o redes de investigación.

\section{Bibliografía}

Young, E.S; Frankenhuis, W.E; Ellis, B.J. 2020. Theory and measurement of environmental unpredictability. Evolution and Human Behavior, 41(6), 550-556 https://doi.org/10.1016/i.evolhumbehav.2020.08.006

Recibido el 13 Dic 2020

Aceptado el 2 feb 2021 